\title{
Porphyra-334, a mycosporine-like amino acid, attenuates UV-induced apoptosis in $\mathrm{HaCaT}$ cells
}

\author{
SUNG-SUK SUH ${ }^{1 *}$ \\ SE KYUNG OH ${ }^{1}$ \\ SUNG GU LEE ${ }^{1,2}$ \\ IL-CHAN KIM ${ }^{1,2}$ \\ SANGHEE KIM ${ }^{1,2 *}$ \\ ${ }^{1}$ Division of Polar Life Sciences \\ Korea Polar Research Institute \\ Incheon, 21990, Republic of Korea \\ ${ }^{2}$ Department of Polar Science \\ University of Science and Technology \\ Incheon, 21990, Republic of Korea
}

Accepted January 13, 2017

Published online March 15, 2017

\begin{abstract}
The main aim of the current research was to study the effect of porphyra-334, one of mycosporine-like amino acids (MAAs), well known as UV-absorbing compounds, on UVinduced apoptosis in human immortalized keratinocyte (HaCaT) cells. Due to their UV-screening capacity and ability to prevent UV-induced DNA damage, MAAs have recently attracted considerable attention in both industry and research in pharmacology. Herein, human $\mathrm{HaCaT}$ cells were used to determine the biological activities of porphyra-334 by various in vitro assays, including proliferation, apoptosis and Western blot assays. The proliferation rate of UV-irradiated $\mathrm{HaCaT}$ cells was significantly decreased compared to the control group. Pretreatment with porphyra-334 markedly attenuated the inhibitory effect of UV and induced a dramatic decrease in the apoptotic rate. Expression of active caspase- 3 protein was increased in response to UV irradiation, while caspase-3 levels were similar between cells treated with porphyra-334 and the non-irradiated control group. Taken together, our data suggest that porphyra-334 inhibits UV-induced apoptosis in HaCaT cells through attenuation of the caspase pathway.
\end{abstract}

Keywords: porphyra-334, apoptosis, cell proliferation, UV irradiation, HaCaT cells

Exposure to environmental ultraviolet radiation induces serious physiological and molecular effects on the cellular metabolism of living organisms, triggering cell damage by directly affecting DNA stability and indirectly through the production of reactive oxygen species (ROS) (1). Several organisms have developed characteristic defense mechanisms to diminish the harmful effects of UV radiation, including DNA damage, by radical quenchers, and antioxidants $(2,3)$. In many photosynthetic marine organisms, one of the most effective UV protection mechanisms is the synthesis of secondary metabolites, such as UV-absorbing mycosporine-like amino acids (MAAs) (4). These compounds are natural compounds found in a wide range of marine organisms and more than 30 different MAAs

\footnotetext{
*Correspondence; email: sung-suk.suh@kopri.re.kr
} 
have been identified $(5,6)$. MAAs have the ability to absorb UV radiation due to their structural characteristics and to dissipate its energy without forming ROS (6, 7). Although a number of recent reports on MAAs in various marine organisms refer mostly to their potential ability to protect from UV, it has been suggested that MAAs also contribute to other cellular functions such as osmotic regulation and antioxidant activity. For example, porphyra-334 exerts potent antioxidant activity and prevents cellular damage caused by UV-induced ROS with free radical scavenging capacity (7). This fact suggests that MAAs can play crucial roles as antioxidant molecules to modulate cellular processes affected by ROS, such as DNA damage and apoptosis. In addition, owing to their effective UV-screening capacity, potential anti-aging effect and cell regenerative ability in human skin cells, MAAs have attracted considerable attention in both industrial and pharmacological fields (8). Chronic exposure to environmental UV radiation leads to functional and structural changes of cells in the skin epidermis and induces photoaging-related processes, such as degradation of collagen fibers, pigmentation and lesions. The UV-induced damage to skin structure leads to significant changes in the expression levels of photoaging-associated genes, such as integrin and pro-collagen $(9,10)$. In fact, a recent report has shown that MAAs from green algae protect skin against UV-induced skin damage through recovery of UV-suppressed expression of elastin and pro-collagen genes (11).

Despite abundant ecological and physiological studies of the functional roles of MAAs on the skin, our understanding of their roles at the molecular level remains poor. Herein, we investigated the physiological and molecular functions of MAAs during apoptosis in UV-irradiated HaCaT cells. A prototype of apoptosis occurring in keratinocytes following UV radiation is the formation of epidermal sunburn cells destined to undergo apoptosis in response to high levels of photosynthetically active radiation (12). This results in activation of cell death pathways through direct interactions with apoptosis-regulating proteins such as the caspase family (13). In the current study, we have demonstrated for the first time that porphyra-334 exerts protective effects against UV-induced apoptosis through attenuation of the caspase pathway in HaCaT cells.

\section{EXPERIMENTAL}

\section{Cell cultures and irradiation}

HaCaT cells were maintained in Dulbecco's modified Eagle's medium (DMEM, Gibco/ Invitrogen, USA) with $10 \%$ fetal bovine serum (FBS) in a $37^{\circ} \mathrm{C}$ humidified incubator containing $5 \% \mathrm{CO}_{2}$. Cells were plated at a density of $0.5 \times 10^{6}$ cells per well in 6 -well plates and were grown overnight. They were incubated with porphyra-334 $\left(0.1 \mathrm{mg} \mathrm{mL}^{-1}\right)$ for 30 min prior to UV irradiation, which was carried out as described previously (11). Cells were exposed to UV light with a Philips Original Home Solarium sun lamp (model HB 406/A, Philips, The Netherlands) equipped with a UV lamp, or were mock-treated, and incubated for $12 \mathrm{~h}$. Three independent experiments of UV-treated HaCaT cells were performed.

\section{MTT assay}

Cells were plated at a density of $1 \times 10^{3}$ cells per well in 96-well plates in triplicate and incubated at $37^{\circ} \mathrm{C}$ with $5 \% \mathrm{CO}_{2}$ in a humidified incubator. Cell viability was measured 
using the 3-(4,5-dimethylthiazol-2-yl)-2,5-diphenyltetrazolium bromide [(MTT)-Cell Titer 96 Aqueous One Solution Cell Proliferation Assay (Promega, USA)] and was analyzed over a period of three days. At every 24-h interval, $20 \mu \mathrm{L}$ MTT was added to detect metabolically active cells. The plate was incubated for $1 \mathrm{~h}$ and cells were analyzed in a Multilabel Counter (Bio-Rad Laboratories, USA).

\section{Annexin V/propidium iodide (PI) staining apoptosis assay}

For the cell apoptosis assay, the Annexin V-FITC apoptosis detection kit (BD Biosciences, USA) was used to assess the apoptotic effect of porphyra-334. For Annexin V staining, HaCaT cells (control, +UV/-porphyra-334 and +UV/+porphyra-334) were harvested by trypsinization, washed twice with cold PBS, and then re-suspended in $100 \mu \mathrm{L} 1 \times$ annexin binding buffer $\left(\sim 1 \times 10^{5}\right.$ cells per $\left.\mathrm{mL}\right)$ containing 5 and $2 \mu \mathrm{L}$ of annexin V-FITC and PI, resp. After cells were incubated for $15 \mathrm{~min}$ at room temperature in the dark, $400 \mu \mathrm{L}$ of $1 \times$ annexin binding buffer was added to each tube and then cells were detected within $1 \mathrm{~h}$ using flow cytometry.

\section{Western blot analysis}

Samples were lysed on ice in RIPA buffer $\left(50 \mathrm{mmol} \mathrm{L}-1\right.$ Tris. $\mathrm{HCl}, \mathrm{pH} 7.5,150 \mathrm{mmol} \mathrm{L}^{-1}$ $\mathrm{NaCl}, 2$ mmol L-1 EDTA, $1 \%$ NP-40, $0.5 \%$ Na-deoxycholate). Total proteins (50 $\mu \mathrm{g}$ ) from each sample were separated on a $4-20 \%$ Criterion $^{\mathrm{TM}}$ Tris $\cdot \mathrm{HCl}$ precast gel (BioRad, USA) and transferred onto nitrocellulose (NC) membranes. The membrane was incubated at $4{ }^{\circ} \mathrm{C}$ overnight with the primary antibody, anti-caspase- 3 antibody (Cell Signaling Technology, USA). After being probed with the secondary antibody, IgG conjugated to horseradish peroxidase (HRP, Santa Cruz Biotechnology, USA), the membrane was developed with enhanced chemiluminescence (Amersham Pharmacia Biotech, UK).

\section{TUNEL assay}

The TUNEL assay was conducted with an in situ cell death detection kit (Roche, USA) per the manufacturer's instructions. Briefly, treated cells were fixed using $2 \%$ paraformaldehyde/PBS ( $\mathrm{pH}=7.4$ ) for $1 \mathrm{~h}$ at room temperature and the fixative was removed by suction, followed by washing in phosphate buffer saline (PBS) twice for 5 minutes. Cells were then incubated with permeabilisation solution for $2 \mathrm{~min}$ in ice and labeled at $37^{\circ} \mathrm{C}$ for $1 \mathrm{~h}$. TUNEL-positive cells were observed by fluorescence microscopy.

\section{Statistical analysis}

Mean values and their standard deviations were calculated from three biological replicates. The statistical significance of the difference between means was tested using oneway ANOVA followed by a Tukey B multi-range test; $p$-values $<0.05$ indicated statistical significance. 


\section{RESULTS AND DISCUSSION}

\section{Effects of MAAs on the growth of HaCaT cells exposed to UV radiation}

To investigate the effects of MAAs on the proliferation rate of cells exposed to UV radiation, we performed the MTT assay on the human keratinocyte cell line, HaCaT, following daily treatment for 24, 48, 72 and $96 \mathrm{~h}$. As shown in Fig. 1a, the proliferation rate of UV-irradiated HaCaT cells was significantly decreased, up to three-fold, compared to nonirradiated cells. Under exposure of UV radiation, human HaCaT cells showed an increase in cell viability after treatment with porphyra-334. Notably, pretreatment with porphyra-334 attenuated the inhibitory effects of UV irradiation to a marked extent, leading to elevated survival rates of $\mathrm{HaCaT}$ cells (up to $\sim 88 \%$ ), compared to non-irradiated cells.

\section{Morphological changes in HaCaT cells treated with porphyra-334 and UV irradiation}

In general, cells undergoing apoptosis display dramatic morphological changes, such as shrinkage and deformation (14). Hence, we further examined whether the morphological deficiencies of UV-induced apoptotic cells were amplified or weakened by porphyra-334. Untreated (control) HaCaT cells were adherent and exhibited a smooth surface and were elongated - irradiation displayed significant shrinkage with irregular and rough forms, along with lower numbers (Fig. 1b). Interestingly, no morphological changes char-

a)

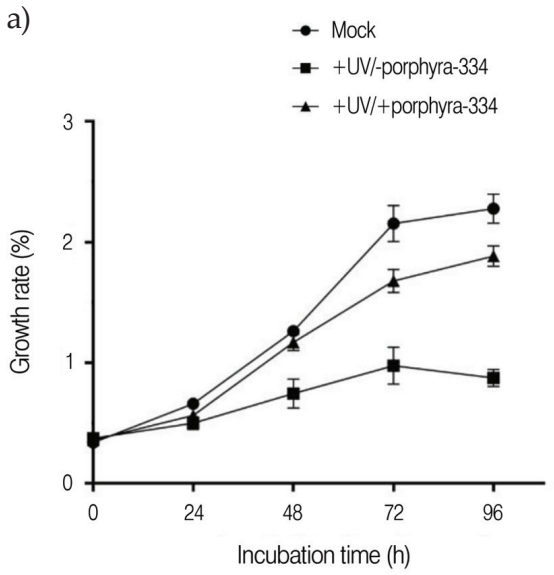

b)

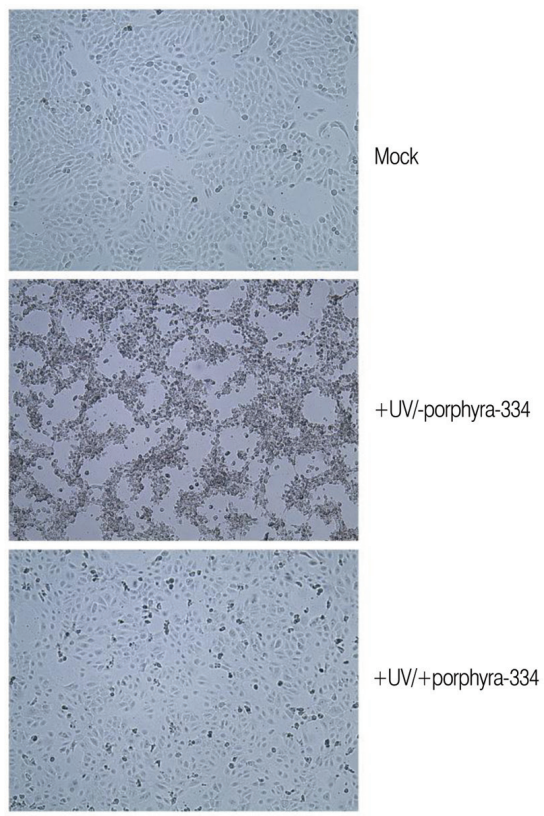

Fig. 1. a) Cell viability and b) morphological change, in UV-exposed HaCaT cells treated with or without porphyra-334. Results are presented as mean \pm SD of three independent experiments. 
acteristic of apoptosis were observed in porphyra-334 treated cells compared to untreated cells, which is consistent with the results of the cell proliferation assay.

\section{Protective effects of $M A A$ s against $U V_{B}$-induced apoptosis and DNA fragmentation through modulation of caspase}

UV-radiation exerts apoptotic effects by causing DNA damage (13). To confirm the protective effects of porphyra-334 on UV-induced HaCaT cells, we performed a flow cytometric analysis to evaluate healthy (AnnexinV-/PI-), early apoptotic (Annexin+/PI-), late apoptotic (AnnexinV+/PI+) and debris (AnnexinV-/PI+) cell populations in the control, UVirradiated, and UV+porphyra-334 treated cells. Annexin V-positive (early and late apoptosis) cells were considered an apoptotic population. As shown in Fig. 2, the apoptotic population was significantly increased in UV-irradiated cells $(23.5 \%)$ compared to the untreated control group $(2.3 \%)$, which was dramatically reduced $(4.0 \%)$ in porphyra-334treated cells. Further, apoptotic cells were observed via TUNEL staining (Fig. 3) designed to detect cells that undergo DNA degradation during the late stages of death. In this assay, most cells were TUNEL positive in the group of +UV/-porphyra-334, implying that UV radiation was able to penetrate into all cells and induce apoptosis through DNA damage. The TUNEL assay measured the effect of porphyra-334 on UV-induced DNA fragmentation and revealed inhibition of DNA fragmentation following addition of porphyra-334;
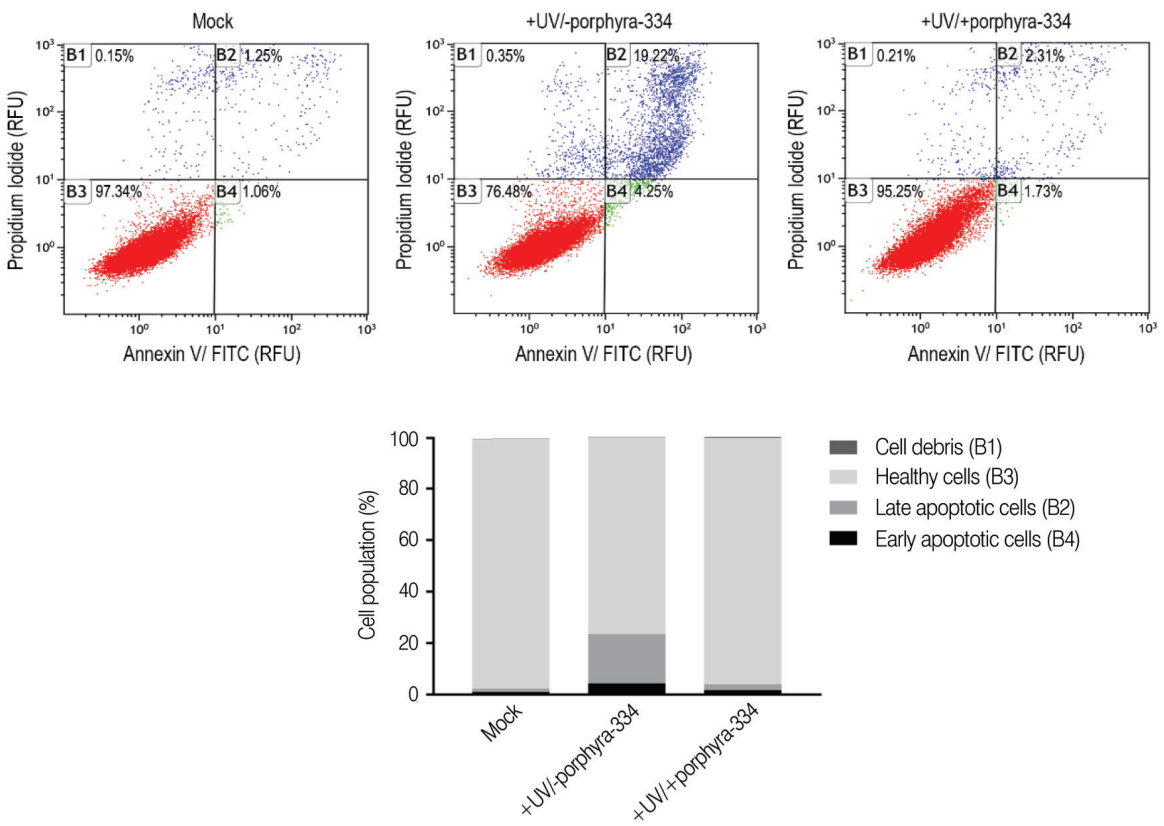

Fig. 2. Porphyra-334 attenuates UV-induced apoptosis. Control and porphyra-334-treated cell populations were examined for Annexin-V expression by flow cytometry analysis. RFU - relative fluorescence units. 


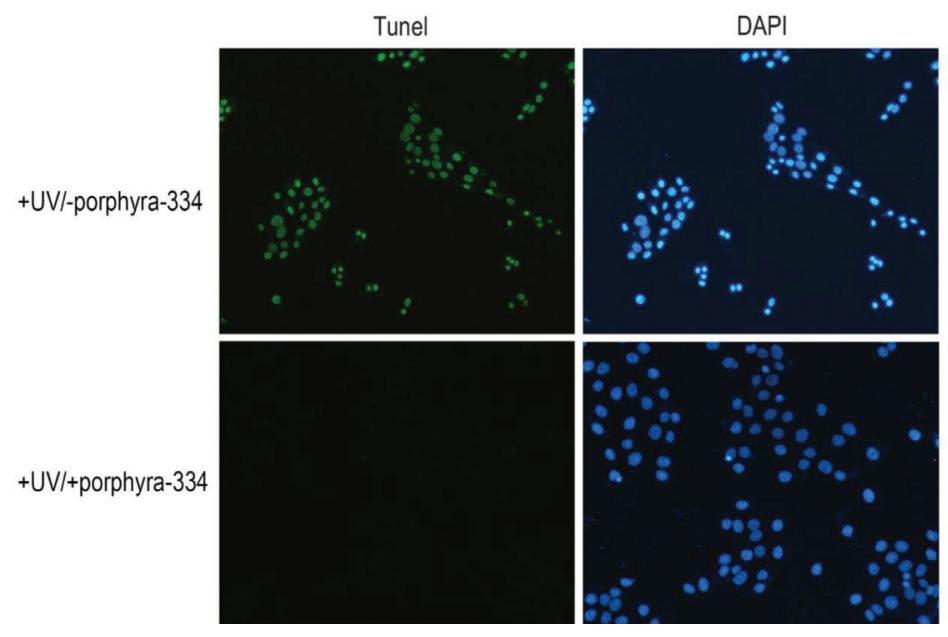

Fig. 3. Inhibition of UV-induced DNA fragmentation by porphyra-334 in HaCaT cells. TUNEL - terminal deoxynucleotidyl transferase dUTP nick end labeling, DAPI - 4',6-diamidino-2-phenylindole (DAPI) staining.

cells from UV-irradiated groups exhibited significant apoptosis whereas porphyra-334 treated cells appeared normal.

Our data suggest that porphyra-334 contributes to the mitigation of UV-induced apoptosis and DNA fragmentation. To ascertain whether porphyra-334 affects the caspase pathway in $\mathrm{HaCaT}$ cells, we measured caspase-3 protein levels. Upon irradiation of $\mathrm{HaCaT}$ cells, activation of caspase-3 (based on the decreased expression of procaspase-3) was clearly observed (Fig. 4). Western blot analysis revealed attenuation of apoptotic signaling in porphyra-334-treated cells. Notably, levels of procaspase-3 and active caspase-3 in porphyra-334 treated cells were restored to $\sim 80 \%$ of control levels, implying that protection of cells from UV damage by porphyra- 334 was partially mediated by suppression of caspase activation.

MAAs are well-known UV light screening/absorbing secondary metabolites produced by a variety of marine organisms, including cyanobacteria and microalgae (4-6). These compounds are colorless and water soluble, composed of cyclohexenone or a cyclohexenimine chromophore conjugated with the nitrogen substituent of an amino acid or amino alcohol (2). The above structural features provide MAA-synthesizing organisms with UV-protective capability, potentially through inducing a considerable decrease in UV-triggered damaging effects with absorption maxima ranging from 310 to $360 \mathrm{~nm}$, such as DNA fragmentation and apoptosis through the modulation of expression levels of apoptosis-related genes, such as p53, Bcl-2 and caspases. Recent reports have shown that MAAs, including porphyra-334, display antioxidant capability with scavenging potential for UVinduced reactive oxygen species, such as superoxide anions, hydroxyl radicals and hydroperoxyl radicals $(2,3)$. Based on this finding, MAAs are suggested to play a protective role in healthy individuals by eliminating ROS and inhibiting apoptosis caused by oxidantmediated cell damage. In addition, various antioxidants inhibit apoptosis by scavenging 

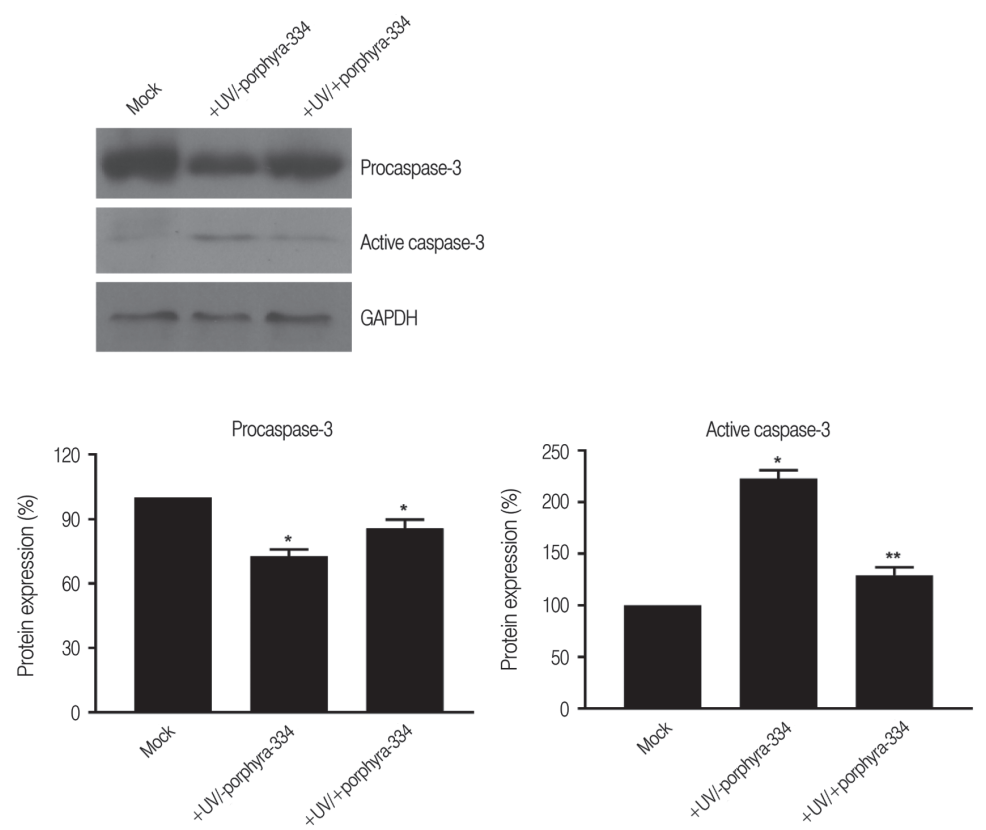

Fig. 4. Western blot analysis for procaspase-3 and active caspase-3 in UV-exposed HaCaT cells treated with or without porphyra-334. Mean \pm SD of three independent experiments. Statistically significant difference between the control and the porphyra-334-treated cells: ${ }^{*} p<0.05$ and ${ }^{* *} p<0.01$.

reactive oxygen species $(2,3)$. In apoptotic cells, procaspase-3 levels are decreased and levels of active forms of caspase- 3 are increased, activating, in turn, endogenous DNases, such as CAD. In our study, elevated active caspase-3 expression in response to UV radiation was significantly decreased upon treatment with porphyra-334. Consistently, data from the TUNEL assay showed that porphyra-334 inhibited DNA fragmentation, the biochemical hallmark of apoptosis, caused by caspase-3-activated endogenous DNases. Although the assay has limitations in sensitivity and specificity due to the interference environments such as DNA condensation and protein environment of DNA, it is a common method for detecting DNA fragmentation that results from apoptotic signaling cascades. Furthermore, the flow cytometry analysis revealed a dramatic reduction in the apoptosis rate in UV-irradiated cells treated with porphyra-334. Taken together, the data clearly suggest that porphyra-334 plays a critical role in protection against the harmful effects of apoptosis provoked by UV radiation-induced DNA damage in HaCaT cells.

\section{CONCLUSIONS}

In the present study, we have demonstrated that porphyra-334 attenuates UV-induced apoptotic cell death in HaCaT cells. The inhibitory effect of porphyra-334 on apoptosis may be attributable to its structural and chemical properties as a mycosporine-like amino acid (MAA). To better understand the protective effect of porphyra-334 against UV radiation, 
we will further examine its functional roles in other human keratinocytes. As a result, our data provide new insights into the use of MAAs for the industrial and pharmacological development of biological sunscreens and antioxidants.

Acknowledgements. - We are grateful to Bio-FD\&C Co. Ltd. of South Korea for providing porphyra-334 used in our work. This work was supported by the Korea Polar Research Institute (KOPRI), under Project PE17100, Republic of Korea.

\section{REFERENCE}

1. S. K. Gao and J. Xu, Effects of solar UV radiation on diurnal photosynthetic performance and growth of Gracilarialemaneiformis (Rhodophyta), Eur. J. Phycol. 43 (2008) 297-307;DOI: 10.1080/09670260801986837.

2. R. Marangoni, D. Paris, D. Melck, L. Fulgentini, G. Colombetti and A. Motta, In vivo NMR metabolic profiling of Fabrea salina reveals sequential defense mechanisms against ultraviolet radiation, Biophys. J. 100 (2011) 215-224; DOI: 10.1016/j.bpj.2010.10.050.

3. M. P. Pessoa, Algae and aquatic macrophytes response to cope with ultraviolet radiation - a review, Emir. J. Food Agric. 24 (2012) 527-545; DOI: 10.9755/ejfa.v24i6.14672.

4. S. Bhatia, A. Garg, K. Sharma, S. Kumar, A. Sharma and A. P. Puohit, Mycosporine and mycosporine-like amino acids: A paramount tool against ultra violet irradiation, Pharmacogn. Rev. 5 (2011) 138-146; DOI: 10.4103/0973-7847.91107.

5. U. Karsten, T. Sawell and C. Wiencke, A survey of the distribution of UV-absorbing substances in the tropical macroalgae, Phycol. Res. 46 (1998) 271-279; DOI: 10.1046/j.1440-1835.1998.00144.x.

6. R. P. Sinha, M. Klisch, A. Groniger and D. P. Häder, Ultraviolet-absorbing/screening substances in cyanobacteria, phytoplankton and macroalgae, J. Photochem. Photobiol. B: Biol. 47 (1998) 83-94; DOI: 10.1016/S1011-1344(98)00198-5.

7. C. Tao, T. Sugawara, S. Maeda, X. Wang and T. Hirata, Antioxidative activities of a mycosporinelike amino acid, porphyra-334, Fish. Sci. 74 (2008) 1166-1172; DOI: 10.1111/j.1444-2906.2008.01636.x.

8. D. Schmid, C. Schürch and F. Zülli, UV-A sunscreen from red algae for protection against premature skin aging, Cosmet. Toilet. Manufact. Worldwide 2004, 139-143.

9. B. M. Steiglitz, J. M. Kreider, E. P. Frankenburg, W. N. Pappano, G. G. Hoffman, J. A. Meganck, X. Liang, M. Höök, S. A. Goldstein and D. S. Greenspan, Procollagen C proteinase enhancer 1 genes are important determinants of the mechanical properties and geometry of bone and the ultrastructure of connective tissues, Mol. Cell Biol. 26 (2006) 238-249; DOI: 10.1128/MCB.26.1.238249.2006.

10. S. Bosset, M. Bonnet-Duquennoy, P. Barré, A. Chalon, K. Lazou, R. Kurfurst, F. Bonté, S. Schnébert, F. Disant, B. Le Varlet and J. F. Nicolas, Decreased expression of keratinocyte $\beta 1$ integrins in chronically sun-exposed skin invivo, Br.J. Dermatol.148(2003)770-778;DOI:10.1046/j.1365-2133.2003.05159.x.

11. S. S. Suh, J. Hwang, M. Park, H. H. Seo, H. S. Kim, J. H. Lee, S. H. Moh and T. K. Lee, Anti-inflammation activities of mycosporine-like amino acids (MAAs) in response to UV radiation suggest potential anti-skin aging activity, Mar. Drugs 12 (2014) 5174-5187; DOI: 10.3390/md12105174.

12. C. H. Lee, S. B. Wu, C. H. Hong, H. S. Yu and Y. H. Wei, Molecular mechanisms of UV-induced apoptosis and its effects on skin residential cells: the implication in UV-based phototherapy, Int. J. Mol. Sci. 14 (2013) 6414-6435; DOI: 10.3390/ijms14036414.

13. T. J. Fan, L. H. Han, R. S. Cong and J. Liang, Caspase family proteases and apoptosis, Acta Biochim. Biophys. (Shanghai) 37 (2005) 719-727; DOI: 10.1111/j.1745-7270.2005.00108.x.

14. A. Saraste and K. Pulkki, Morphologic and biochemical hallmarks of apoptosis, Cardiovasc. Res. 45 (2000) 528-537; DOI: 10.1016/S0008-6363(99)00384-3. 